\title{
Thyroid Doses and Skin Contaminations of Radioiodine
}

Kalevi Kairemo ${ }^{1,2^{*}}$

${ }^{1}$ Department of Molecular Radiotherapy \& Nuclear Medicine, Docrates Cancer Center, Fl-00180 Helsinki, Finland

${ }^{2}$ Department of Nuclear Medicine, The University of Texas MD Anderson Cancer Center, Houston, TX 77030, USA

*Corresponding author: Kalevi Kairemo, Department of Molecular Radiotherapy \& Nuclear Medicine, Docrates Cancer Center, Saukonpaadenranta 2, FI-00180 Helsinki, Finland, Tel: +358 10773 2000; Fax: +358 10773 2099; E-mail: kalevi.kairemo@docrates.com

Received date: Aug 31, 2015, Accepted date: Sep 10, 2015, Publication date: Sep 14, 2015

Copyright: ( 2015 Kairemo K. This is an open-access article distributed under the terms of the Creative Commons Attribution License; which permits unrestricted use; distribution; and reproduction in any medium; provided the original author and source are credited.

\begin{abstract}
Radioiodine treatment, using iodine $\mathrm{I}-131$, is one of the oldest clinical radionuclide therapy methods, and it is widely spread and currently used in the treatment of both thyreotoxicosis and thyroid cancer. The use of iodine may cause skin contaminations which may be a radiobiologic hazard and diagnostic problem.
\end{abstract}

Reported hazards in the radiopharmaceutical production of $\mathrm{l}-131$ for therapy purposes are rare, but they might cause therapeutic approaches due to radioiodine uptake in the thyroid and destruction of thyroid tissue (mean beta range, $0.8 \mathrm{~mm}$ ).

In this work a skin contamination causing a radioiodine thyroid uptake has been reported, together with thyroid cancer patients of receiving therapeutic doses of I-131 having skin contaminations from exudates.

These skin contaminations were quantificated, dose calculations were performed based on serial gamma detector and quantitative whole body gamma imaging, including SPECT/CT. For serial gamma imaging, thyroid retention and whole clearance after oral administration, rhTSH stimulation and thyroxin withdrawal give different results and therefore serial uptake estimates and time-activity curves are essential parameters for dose evaluation. Similarly, accidental subcutaneous administration for calculating thyroid uptake requires serial measurement as well.

In these patients, the skin contaminations did not cause any potential hazard to the environment nor to the hospital even though potential risks have been reported in the early days. Skin doses due to contaminations varied from 0.64 to $2.7 \mathrm{~Gy}$. In the radiopharmaceutical production accidental skin contamination did not cause any harmful thyroid dose ( $80 \mathrm{mGy}$ ) due to rapid cleaning actions.

Quantitative gamma imaging together with activity calibrated detectors is the prerequisite for appropriate dose calculations when routes of administration differ from clinical routine.

Keywords: Skin contamination; Radioiodine uptake; I-131; Radiopharmaceutical preparation; Thyroid dose; Radioiodine therapy

\section{Introduction}

Skin contaminations are rare in radiopharmaceutical production, or at least they have not been reported in the scientific literature.

It is important for radiation safety purposes to understand the severity of the possible contaminations, so that appropriate therapeutic approaches can be made. Additionally, in order to avoid unnecessary therapeutic interventions it is extremely important to properly distinguish false-positive sites of radioiodine (I-131) localization. The physical half-life of iodine is suitable for radionuclide treatment $\left(\mathrm{T}_{1 / 2}=8.03\right.$ days $)$, but the conditions are different in tyreotoxicosis and cancer treatments because of whole body effective half-lifes $\left(\mathrm{T}_{1 / 2 \text {,eff }} \approx 4-7\right.$ days $)$ and differentiated thyroid cancer $\left(\mathrm{T}_{1 / 2 \text {,eff }} \approx 0.5-5\right.$ days). The beta energy effects in body are characterized by beta decay, $\mathrm{E}_{\beta}=606 \mathrm{keV}$ (89\%), mean energy $190 \mathrm{keV}$, making maximum energy in tissue $2 \mathrm{~mm}$, mean energy $0,6 \mathrm{~mm}$. Radioiodine has gamma energies which make imaging possible: $\mathrm{E}_{\gamma}=364 \mathrm{keV}(89 \%), 637 \mathrm{keV}$ (7\%) ja 282
$\mathrm{keV}(6 \%)$. This part of decay generates the radiation protection rules, even patient isolation in clinical practice.

In the literature, skin contaminations in radioiodine imaging of thyroid cancer metastases have been reported [1,2]. Reported cases of iodine-131 accumulation in the literature consist of contamination in styling hair with saliva, contamination in neck area while rolling during sleep and, contaminated chewing gum [2], handkerchiefs in pocket [3], and neck and breast region due to a contaminated bra [4].

Even a systemic analysis of skin artefacts has been reported [5]. In this study, 22 foci that simulated lung metastasis on 11 local thyroid and whole body scans were proven not to be metastasis by reimaging after cleaning (15 foci), or were strongly suspected to be due to external contamination because of the features of other images ( 7 foci) [5]. Potential hazard calculated for environment have been published as well, and nursing staff and visitor may be exposed to moderate doses, if appropriate attention is not paid to hospital radiation hygiene [1].

This a report of a case who was referred to our Institution for clinical dose evaluation after radioiodine skin contamination and three 
additional cases with skin contaminations associated with radioiodine treatment are reported.

\section{Case Reports}

\section{Case 1}

A 36-year-old female laboratory technician was preparing a therapeutic radioiodine dose in a GMP manufacturing area. Part of her protection was to use gloves in duplicate, inner gloves (Ansell TNT Blue nitrile gloves (Ansell Healthcare, Iselin, NJ)) and outer gloves were Semperguard latex powder-free disposable gloves (Semperit Technische Produkte GmbH, Vienna, Austria). After finishing the work a small cut was observed in the inner glove of the right hand but the outer glove was tearless.

After finishing the work both hands were controlled with monitor (Berthold LB $1043 \mathrm{~B}^{\circ}$, hand-foot-monitor; Berthold Technologies $\mathrm{GmbH}, \mathrm{Bad}$ Wildbad, Germany) and a contamination was measured.

The hands were washed carefully multiple times but a minor skin contamination remained, the activity fell down from 2800 cps to 1400 cps. Sequential measurements and washings did not anymore decrease the activity.

On the following day the activity in the thyroid was $450 \mathrm{cps}$ as was measured on the hand with a handheld detector (MINI Series 900 mini-monitor (MFG012) equipped Mini-Instruments gamma probe type 41; ThermoScientific, Burnham-on-Crouch; UK). The thyroid activity was approximately $120 \mathrm{kBq}$ (based on the gamma probe calibration) on day 1 .

The half-life approximately in the hand contamination was $16-20$ hrs based on hald-held detector measurements. Because of a slow accumulation in the thyroid due to subcutaneous route of administration, further serial measurements were carried out.

At 22 days, whole body (WB) imaging was performed with a dual head gamma camera (Siemens Symbia T2) using a high energy collimator. The whole body imaging took 80 minutes and a weak uptake in the thyroid was seen, but no other organs were visualized, such as salivary glands or stomach (parietal cells).

The activity in the thyroid was now $3.1 \mathrm{kBq}$ based on quantitative geometric mean images. The activity in the thyroid corresponded $1.2 \%$ of the whole body activity. The whole body images are shown in Figure 1.

Additionally spot images, using 30 minute frames were taken placing hands on the collimator but no activity in the hands was observed Figure 2, neither on palmar nor volar images.

In this accident there were two organs which were affected. The skin locally received the highest dose, the absolute dose can be estimated only from the thyroid dose. At the beginning, the skin was occasionally irritated, dry and squamous, but did not demonstrate any ulcerations. There we no symptoms after two weeks, and the activity had totally disappeared as demonstrated by the scintigraphy.

In this case, if the maximal thyroid activity was $120 \mathrm{kBq}$ on day 1. This would give for the thyroid an approximate half-life of less than 4 days, because the actual uptake on day 22 was $3.1 \mathrm{kBq}$ based on quantitative imaging. This would give (assuming 35\% uptake) $0.08 \mathrm{~Gy}$ for a thyroid dose and effective whole body dose $7 \mathrm{mSv}$.

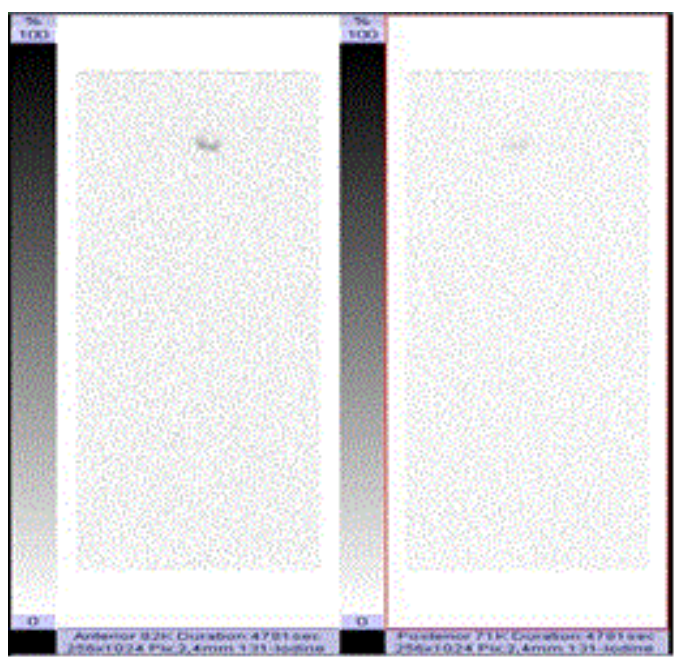

Figure 1: Radioiodine imaging of after accidental radioiodine (I-131) skin contamination at 22 days. WB imaging reveals only minor activity in the thyroid ( $1.2 \%$ of the whole body activity).

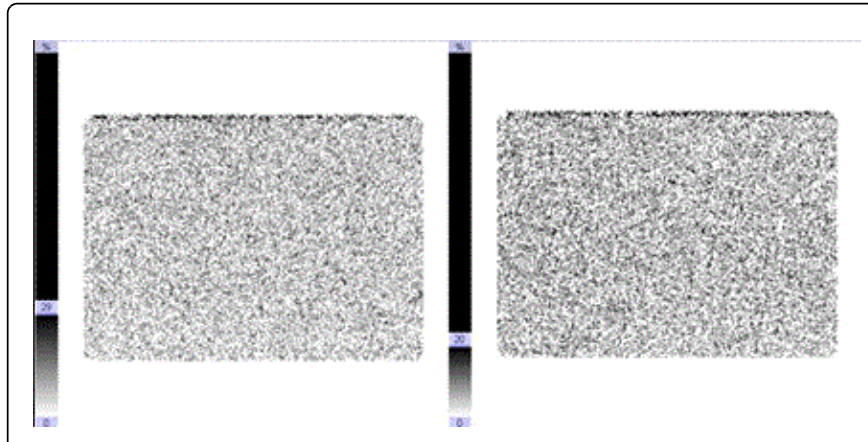

Figure 2: Radioiodine imaging of after accidental radioiodine (I-131) skin contamination at 22 days. Spot images do reveal any activity in the contaminated are $(0.000 \%$ of the whole body activity).

\section{Cases 2-4}

Case 2: Case 2 is a 45-year-old woman papillary with thyroid cancer treated initially with $1.056 \mathrm{GBq}$ of radioiodine using rTSH stimulation. Her thyroid gland was removed together with enlarged jugular lymph nodes on the right 6 months earlier. The pathologic staging was pT2N0M0, histology was papillary carcinoma of the thyroid, with no metastases in the regional lymph nodes, and no invasion to the thyroid capsule.

Whole body imaging with the thyroid ablation dose demonstrates a quite large thyroid residue in both lobes and in the midline, corresponding $12 \%$ of $\mathrm{WB}$ activity at 2 days. Besides, a rather large thyroid residue ( $12 \%$ of the whole body activity at 2 days), the whole body image demonstrates a weak uptake laterally in the left calf $(0.08 \%$ geometric mean of the WB uptake) (Figure 3). 
This skin contamination is better seen in the PA view (Figure 3). SPECT/CT demonstrated that the thyroid activity lied predominantly in the left lobe, but there was residual activity in the right lobe as well. The midline activity was in the thyreoglossal duct. There was minor activity in the right maxillary cavity, but this was considered physiological.

Hand held-detector measurements gave as dose rate at $0 \mathrm{hrs} 68.5$ $\mu \mathrm{Sv} / \mathrm{h}$, at $24 \mathrm{hrs} 9.5 \mu \mathrm{Sv} / \mathrm{h}$ and at $45 \mathrm{hrs} 5 \mu \mathrm{Sv} / \mathrm{h}$ from 1 meter distance.

The skin contamination was $0.08 \%$ of the WB at $45 \mathrm{hrs}$. This activity corresponded with $62 \mathrm{kBq}$ activity. The absorbed skin dose was 0.96 Gy using Monte Carlo code calculation [6].

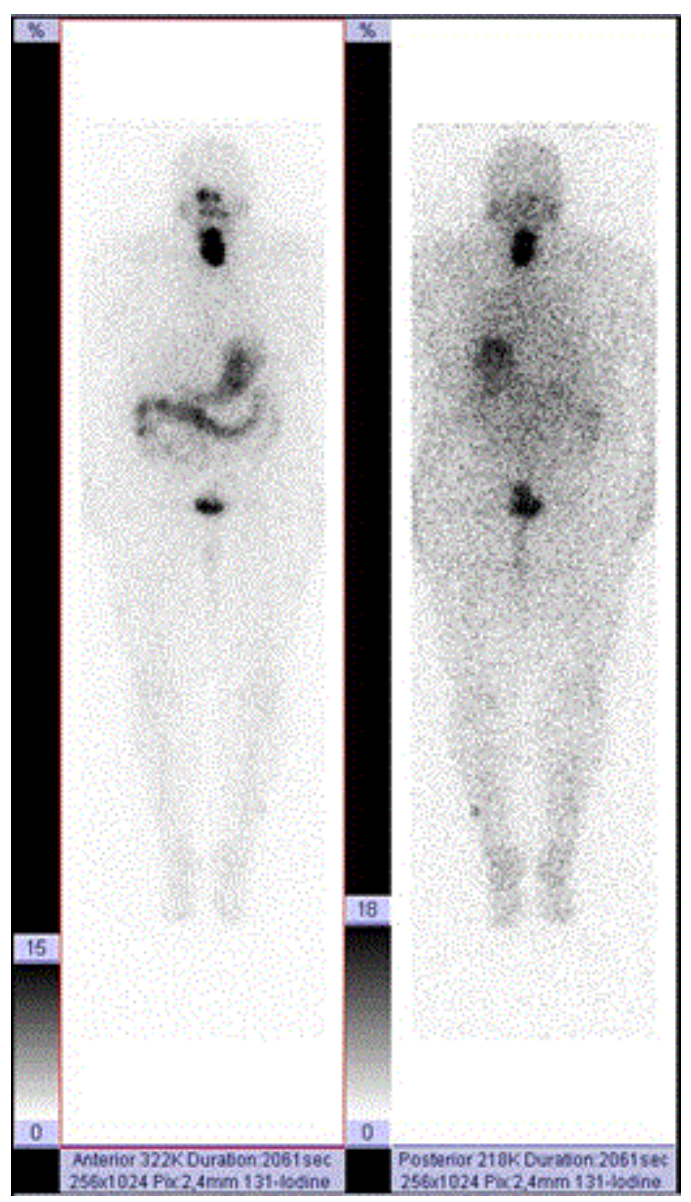

Figure 3: Radioiodine imaging of thyroid cancer metastases using rTSH stimulation, i.m. (Thyrogen) 1 and 2 days before WB imaging at $45 \mathrm{~h}$ p.i.. The administered oral acivity was $1.056 \mathrm{GBq}$. This patient had papillary carcinoma of thyroid gland pT2N0M0, no invasion to capsule or lymph nodes. Whole body imaging demonstrates a quite large thyroid residue in both lobes and in the midline ( $12 \%$ of WB activity at $2 \mathrm{~d}$ ), but no suspicious foci outside the neck region in planar imaging. Normal physiologic activity is seen in nasopharynx, salivary glands, stomach, GI tract and urinary bladder. A minor skin contamination is seen laterally in the left calf.

Case 3: Case 3 is a 53-year-old woman with follicular thyroid cancer treated initially with $3.632 \mathrm{GBq}$ using thyroxin withdrawal for 4 weeks.
She had been operated 12 months earlier for follicular thyroic cancer, pT1NxMx, stage I and she come to our institution for ablation therapy.

The whole body images with the thyroid ablation dose demonstrates a small thyroid residue (3\% of the whole body activity at 3 days), and weak uptake dorsally uptake in the left heel $(0.18 \%$ geometric mean of the WB uptake). This skin contamination is better seen in the PA view (Figure 4). A lateral spot image shows the exact location (Figure 5). SPECT/CT confirmed that there was only thyroid involvement in the right lobe, about $2 \frac{1}{2} \mathrm{~cm}$ in size, no neck, lung nor mediastinal uptakes.

Hand held-detector measurements were as follows: at $0 \mathrm{hrs} 166$ $\mu \mathrm{Sv} / \mathrm{h}$, at $24 \mathrm{hrs} 40 \mu \mathrm{Sv} / \mathrm{h}$, at $48 \mathrm{hrs} 12 \mu \mathrm{Sv} / \mathrm{h}$ and at $70 \mathrm{hrs} 5 \mu \mathrm{Sv} / \mathrm{h}$ from 1 meter distance. The skin contamination was $0.18 \%$ of the WB at 70 hrs. This activity corresponded with $197 \mathrm{kBq}$ activity. The absorbed skin dose was 2.7 Gy using Monte Carlo code calculation [6].

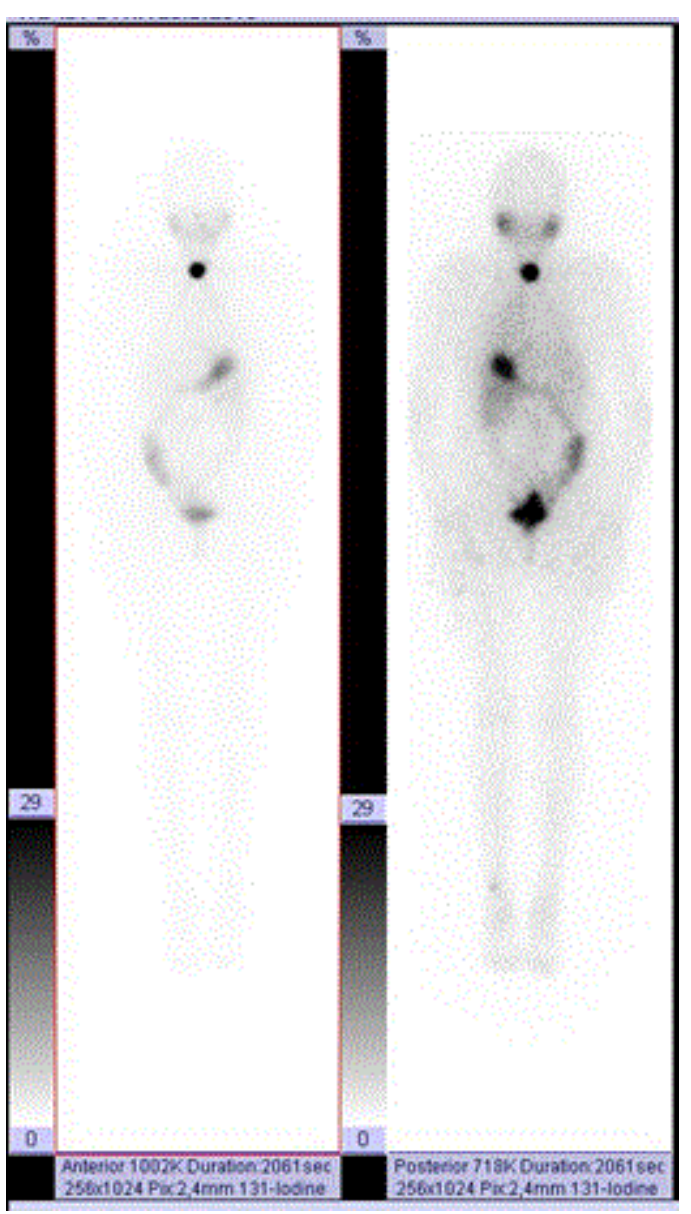

Figure 4: Radioiodine imaging of thyroid cancer metastases using thyroxin withdrawal for 4 weeks. WB imaging was performed at 70 h p.i.. The administered oral acivity was $3.632 \mathrm{GBq}$. This patient had follicular carcinoma of thyroid gland pT1NxMx, no invasion to capsule or lymph nodes. Whole body imaging demonstrates a small thyroid residue in the right lobe ( $3 \%$ of $\mathrm{WB}$ activity at $3 \mathrm{~d}$ ), and no suspicious foci outside the neck region in planar imaging. Normal physiologic activity is seen in salivary glands, stomach, GI tract and urinary bladder. A minor skin contamination is seen in the left ankle. 
Citation: Kairemo K (2015) Thyroid Doses and Skin Contaminations of Radioiodine. J Nucl Med Radiat Ther 6: 244. doi: $10.4172 / 2155-9619.1000244$

Page 4 of 5

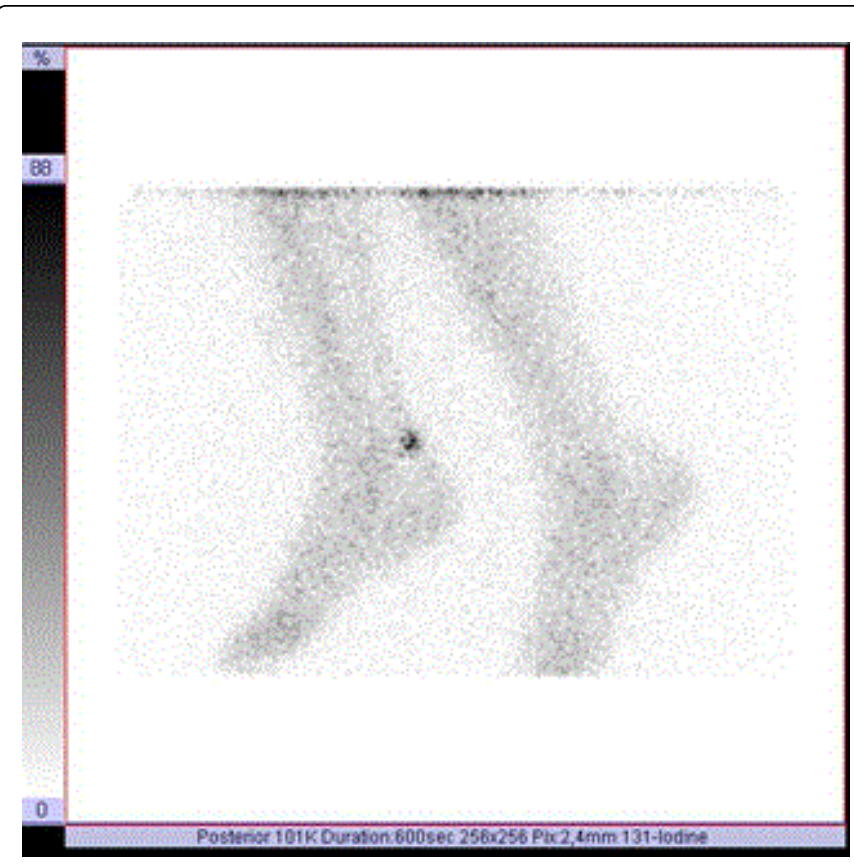

Figure 5: Radioiodine imaging of thyroid cancer metastases using thyroxin withdrawal for 4 weeks. WB imaging was performed at 70 h p.i.. The administered oral acivity was $3.632 \mathrm{GBq}$. (Side view, lower extremities distally). There is a minor uptake above the left ankle. Side view reveals a skin contamination dorsally above distal achilles tendon, the activity was $0.18 \%$ of whole body activity.

Case 4: Case 4 is a 75-year-old man with Stage IV metastasized thyroid cancer. At the time of diagnosis, there were already metastases in the lungs and liver, clinical staging T4N1bM1. Originally, the patient was operated with thyroidectomy. But after developing a local relapse the patient was inoperable, and radioiodine treatment was recommended to be given as palliation, and therefore this patient was sent to our Institution. The biopsy revealed that this patient had a lowdifferentiated follicular carcinoma from papillary carcinoma with invasive growth and perineural growth, and it had a desmoplastic component. In the diagnostic CT, besides a left lobe thyroid cancer and loco regional neck lymph node metastases, this patient had lung metastases, retroperitoneal lymph node metastases and skeletal lesions.

Radioiodine imaging in this patient with Stage IV metastasized thyroid cancer was performed as a part of palliative treatment. The orally given activity was $5.082 \mathrm{GBq}$ using i.m. rTSH stimulation 1 and 2 days before oral administration. The whole body images with the palliative dose demonstrate a small thyroid residue $(2.9 \%$ of the whole body activity at $2 \& 6$ days), diffuse lung metastases, and multiple skeletal metastases (thorax, spine, pelvis) and contaminations in clothing especially in the $6 \mathrm{~d}$ image laterally on the left and in the skin on the right thigh $(0.18 \%$ geometric mean of the WB uptake). SPECT/CT located the iodine activity in the neck lymph nodes on the left, in the left lobe caudally with invasion to surrounding tissues deviating trachea.

Detector measurements gave $127 \mu \mathrm{Sv} / \mathrm{h}(0 \mathrm{~h}) ; 86 \mu \mathrm{Sv} / \mathrm{h}(24 \mathrm{~h}) ; 96$ $\mu \mathrm{Sv} / \mathrm{h}(45 \mathrm{~h}) ; 19 \mu \mathrm{Sv} / \mathrm{h}(116 \mathrm{~h}) ; 8.4 \mu \mathrm{Sv} / \mathrm{h}(139 \mathrm{~h})$. The skin uptake corresponded with $62 \mathrm{kBq}$ activity. The absorbed skin dose was 0.64 Gy using Monte Carlo code calculation [6].

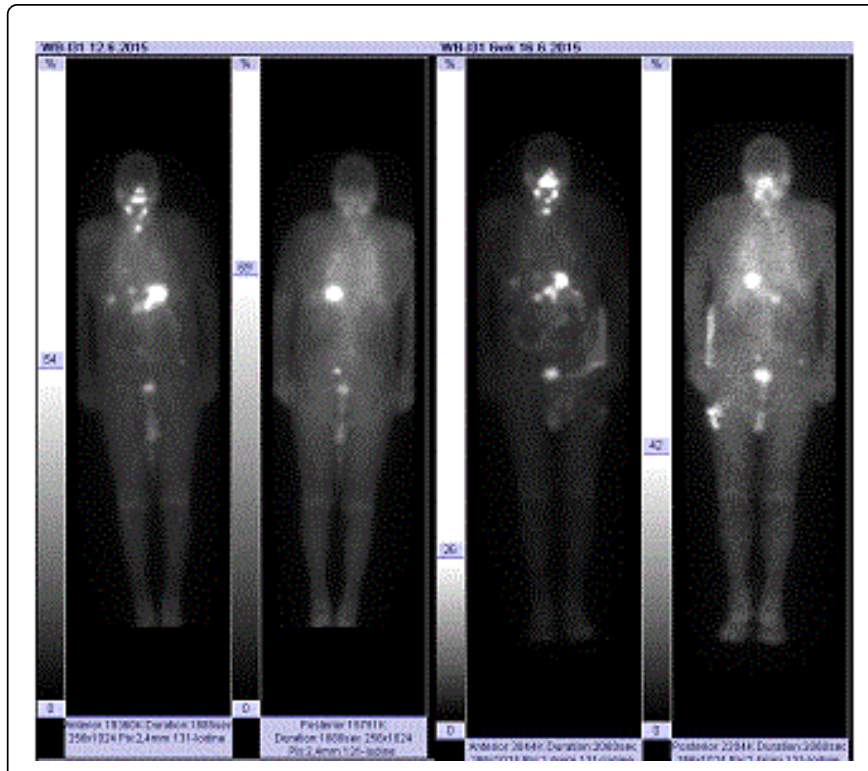

Figure 6: Radioiodine imaging of thyroid cancer metastases using rTSH stimulation, i.m. (Thyrogen ) 1 and 2 days before. WB imagings were performed at 45 and $119 \mathrm{~h}$ p.i.. The administered oral activity was $5.082 \mathrm{GBq}$. This patient had Stage IV metastasized thyroid cancer. Whole body imaging demonstrates a small thyroid residue, diffuse lung uptakes bilaterally, multiple skeletal uptakes in thorax, spine and pelvis. A strong normal physiologic activity is seen in stomach, normal physiological activity in salivary glands and urinary bladder. Contaminations in clothing are seen on the left, especially in the $6 \mathrm{~d}$ image.

\section{Discussion}

Radioiodine is actively taken up by thyroid and the activity is increased at least up to 4 days. The normal half-life in thyroid tissue is 5-7 days. Anyway, the thyroid dose was low in the skin contamination case, $80 \mathrm{mGy}$, based on quantitive gamma imaging, 80 minutes scanning on day 22 . The quantitative uptake in the thyroid was 3.1 $\mathrm{kBq}$, and the uptake was estimated as $35 \%$ (normal range $20-45 \%$ ). The hand-detector measurements gave as thyroid uptake quantitively 120 $\mathrm{kBq}$. Then the half-life would be less than 4 days which is outside the normal range. The real assumption is that the thyroid uptake as measured by hand held detectors is over-estimated. With normal 6 day half-life the maximum uptake in the thyroid would have been less than $40 \mathrm{kBq}$, i.e. more than 3 -fold over-estimation in the original thyroid activity. This leads to the conclusion, that quantitative serial imaging is an essential part of dose quantifications.

In the radioiodine therapy of to achieve euthyreosis in hyperfunctioning thyroid, the target dose is $400 \mathrm{~Gy}$, i.e. 5000 -fold as seen here. Thus the thyroid dose did not cause any damage in this accident.

Radioiodine, because non-physiologic I-131 uptake detected on images is usually interpreted as suggesting functioning thyroid metastases. However, extra-thyroidal I-131 accumulation does not always imply thyroid cancer metastases and these phenomena have been reported on many occasions, including various non-thyroidal 
Page 5 of 5

neoplasms, and contamination by body secretions, e.g. urinary contaminations (Figures 3-6).

In order to avoid unnecessary therapeutic interventions, false positive contamination sources should be clarified by careful examination of patients and further images when necessary (Figure 5). False positive uptakes have also been listed systematically in the literature [7], including a number of potential pitfalls in the interpretation of this scan that could lead to a false-positive diagnosis of cancer [8]. False-positive localisations of radioiodine due to body secretions, pathologic exudate, activity in nonlactating breast, salivary gland inflammation, ovarian teratoma [9] and chest [10]. Some of these false-positive can be ruled out easily by removing of clothes, removing of nozzle in patients with tracheostomy, or by ingestion of water in subjects with abnormal mediastinal hyperfixation [9].

For dosimetry, two parameters are needed; uptake in organ and mean residence times. To make this precisely, serial quantitative measurements are required to understand pharmacokinetics in various organ systems $[11,12]$.

In these patients, imaged with radioiodine hand-held detector measurements explained the time course and one whole body combined with SPECT/CT gave an estimate about the dosimetry. In the thyroid cancer patients (case 2 and case 3), combining kinetics with distribution was more accurate while hand-held measurement could be calibrated with the quantitative gamma camera data on the same day.

However, in the case 1, the hand-held detector measurements were performed elsewhere and precise activity calibration was not possible. Therefore, also the calculations were performed separately. Even, the time-activity course is somewhat indefinite for subcutaneous radioiodine uptake (I-131), maximum may not be achieved by 4 days. This explains the possible discrepancy in the calculated thyroid doses based on quantitative imaging as compared to detector measurements.

The skin contamination did not here exceed, hand activity of those measured in thyroid cancer patients [1]. Skin dose rate conversion factors after contamination with radiopharmaceuticals, including I-131, are presented in the literature [6]. In our case, the skin doses were low, i.e. 0.64, 0.96 and $2.7 \mathrm{mSv}$ as calculated using the Monte Carlo particle transport code, i.e approximately $1.4 \mathrm{mGyh}^{-1} \mathrm{kBq}^{-1}[9]$.

A skin contamination was reported in therapy dose radiopharmaceutical preparation. Skin contaminations are also common among radioiodine therapy patients, and three more are reported here. Minimum prerequisite for dose calculation are quantitative gamma camera measurements combined with serial handheld detector measurements which are synchronized with activity calibration. Otherwise, the results remain elusive as the case, where measurements were performed at multiple institutions.

\section{References}

1. Ibis E, Wilson CR, Collier BD, Akansel G, Isitman AT, et al. (1992) Iodine-131 contamination from thyroid cancer patients. J Nucl Med 33: 2110-2115.

2. Ozcan Kara P, Sari O, Gedik GK, Kocak I, Kaya B (2011) An interesting contamination artifact appearing in I131- whole-body imaging after ablative therapy. Rev Esp Med Nucl 30: 36-37.

3. Pochis WT, Krasnow AZ, Isitman AT, Cerletty JM, Kir KM, et al. (1990) The radioactive handkerchief sign. A contamination artifact in I-131 imaging for metastatic thyroid carcinoma. Clin Nucl Med 15: 491-494.

4. Ozguven M, Ilgan S, Arslan N, Karacalioglu AO, Yuksel D, et al. (2004) Unusual patterns of I-131 contamination. Ann Nucl Med 18: 271-274.

5. Bakheet S, Hammami MM (1993) Spurious lung metastases on radioiodine thyroid and whole body imaging. Clin Nucl Med 18: 307-312.

6. Covens P, Berus D, Cavaliers V, Struelens L, Vanhavere F, Verellen D (2013) Skin dose rate conversion factors after contamination with radiopharmaceuticals: influence of contamination area, epidermal thickness and percutaneous absorption. J Radiat Prot 33: 381-393.

7. McDougall IR (1995) Whole-body scintigraphy with radioiodine-131. A comprehensive list of false-positives with some examples. Clin Nucl Med 20: 869-875.

8. Oh JR, Ahn BC (2012) False-positive uptake on radioiodine whole-body scintigraphy: physiologic and pathologic variants unrelated to thyroid cancer. Am J Nucl Med Mol Imaging 2: 362-385.

9. Rudoni S, Toubeau M, Mansuy S, Vaillant G, Verges B, et al. (1997) Faux positifs scintigraphiques à l'iode 131 dans la surveillancedes cancers thyroïdiens différenciés. [False positive scintigraphic images in the surveillance of differentiated thyroid cancer]s. Ann Endocrinol (Paris) 58: 399-407.

10. Garcia Alonso MP, Balsa Breton MA, Paniagua Correa C, Castillejos Rodriguez L, Penin Gonzalez FJ, et al. (2011) Captacion toracica de 131I en el seguimento del cancer diferenciado te tiroides. Rev Esp Med Nucl 30: 24-28.

11. Kairemo K, Kangasmaki A (2013) 4D SPECT/CT acquisition for 3D dose calculation and dose planning in 177Lu-peptide receptor radionuclide therapy: applications for clinical routine. Recent Results Cancer Res 194: 537-550.

12. Heiskanen T, Heiskanen T, Kairemo K (2009) Development of a PBPK model for monoclonal antibodies and simulation of human and mice PBPK of a radiolabelled monoclonal antibody. Curr Pharm Des 15: 988-1007. 